\title{
Concurrent Sexual Partnerships Among Men in the United States
}

\author{
| Adaora A. Adimora, MD, MPH, Victor J. Schoenbach, PhD, and Irene A. Doherty, PhD
}

Population-level parameters of sexual behavior are important determinants of HIV transmission. ${ }^{1}$ Concurrent sexual partnerships (sexual partnerships that overlap in time) have emerged as potentially important factors in the spread of HIV and other sexually transmitted infections. ${ }^{2-4}$ Concurrent sexual partnerships permit faster dissemination of infection through a network than would the same number of new, sequential sexual partnerships, ${ }^{2}$ especially during acute HIV infection when transmission probability is greatest. ${ }^{5}$ Once someone is infected with HIV by a sexual partner, transmission to another (concurrent) sexual partner can occur without the delay involved in ending the first partnership and beginning the second. The potential role of concurrent sexual partnerships in promoting transmission of HIV and other sexually transmitted infections has been shown in mathematical models ${ }^{2,6}$ and suggested by associations with transmission of chlamydia ${ }^{7}$ and syphilis. ${ }^{8}$ Having a sexual partner with concurrent sexual partnerships has emerged as an independent risk factor for acquisition of heterosexually transmitted HIV infection among African Americans. ${ }^{9}$

Concurrent sexual partnerships could contribute to heterosexual transmission of HIV and other sexually transmitted infections in the United States. We previously reported a 5-year concurrency prevalence of 12\% among women who participated in the 1995 National Survey of Family Growth (NSFG), ${ }^{10}$ but this survey did not include men, and no published reports document the extent of concurrent sexual partnerships among American men. To determine the prevalence and behavioral and demographic correlates of concurrency among men in the United States, we analyzed data from the 2002 NSFG.

\section{METHODS}

The NSFG is conducted periodically by the National Center for Health Statistics (NCHS)

Objectives. We sought to determine the prevalence, distribution, and correlates of US men's involvement in concurrent sexual partnerships, a sexual network pattern that speeds population dissemination of HIV.

Methods. For this analysis, we compared sexual partnership dates of 4928 male respondents in the 2002 National Survey of Family Growth to determine the prevalence of concurrent sexual partnerships and evaluated associations between concurrency and demographic risk characteristics.

Results. Approximately $11 \%$ of men had concurrent sexual partnerships during the preceding year. Concurrency was associated with being unmarried (odds ratio $[\mathrm{OR}]=4.59 ; 95 \%$ confidence interval $[\mathrm{Cl}]=2.54,8.29)$, non-Hispanic Black $(\mathrm{OR}=2.56 ; 95 \% \mathrm{Cl}=1.61,4.07)$ or Hispanic ( $\mathrm{OR}=2.25 ; 95 \% \mathrm{Cl}=1.32,3.85)$ race/ethnicity, and incarceration during the past year $(\mathrm{OR}=2.10 ; 95 \% \mathrm{Cl}=1.18,3.74)$. Men with concurrent sexual partnerships were also more likely to report drug or alcohol intoxication during sexual intercourse $(\mathrm{OR}=2.10 ; 95 \% \mathrm{Cl}=1.37,3.21)$, nonmonogamous female sexual partners $(\mathrm{OR}=6.11 ; 95 \% \mathrm{Cl}=4.10,9.11)$, and history of sexual intercourse with a man $(\mathrm{OR}=1.93 ; 95 \% \mathrm{Cl}=1.09,3.42)$, than those without concurrent partnerships.

Conclusions. The higher concurrency prevalence in various groups, dense sexual networks, and mixing between high-risk subpopulations and the general population may be important factors in the US epidemic of heterosexual HIV infection. (Am J Public Health. 2007;97:2230-2237. doi:10.2105/AJPH.2006.099069)

of the Centers for Disease Control and Prevention to collect data on reproductive behavior and experience in the United States. ${ }^{11}$ Data collection for Cycle 6 of the survey took place from March 2002 through March 2003.

\section{Sample}

The NSFG Cycle 6 used area probability sampling and a complex, multistage, stratified design to obtain a national probability sample that represented the household population of persons aged 15 to 44 years in the 50 states and the District of Columbia. ${ }^{12}$ People living away from home in college or university dormitories, fraternities, and sororities were listed in their household of usual residence and eligible for interview if selected. Blacks, Hispanics, women, and people aged 15 to 19 years and 20 to 24 years were oversampled. The overall $78 \%$ response rate yielded 4928 completed interviews of men. The NCHS provides sample weights adjusted for subsampling, nonlocation, nonresponse, and census estimates of the US population.

\section{Interview Technique}

Female interviewers administered the survey using computer-assisted personal interview (CAPI) technology; audio computer-assisted self-interviewing (ACASI) was used for especially sensitive questions at the end of the interview. For example, the CAPI survey queried each respondent concerning the date (month and year) of first and last sexual intercourse with his current wife or female cohabiting partner and his 3 most recent female sexual partners during the preceding 12 months. The ACASI section of the interview asked the respondent (1) whether he had ever had oral or anal sexual intercourse with a man and (2) how many men he had sexual intercourse with during the past 12 months, but did not ascertain the dates of his relationships with men or women.

\section{Definition of Concurrent Sexual Partnerships}

We identified concurrent sexual partnerships as follows: partnerships of each respondent 
whose CAPI questionnaire provided dates of first and last sexual intercourse with at least 2 female sexual partners during the preceding 12 months were ordered sequentially by date of first sexual intercourse, and the dates of first and last sexual intercourse were compared across all female sexual partnerships. A female sexual partnership was considered concurrent with another female sexual partnership if the date of first sexual intercourse with 1 partner occurred before the date of last intercourse with an earlier sexual partner. A female sexual partnership was considered concurrent with a male sexual partnership if a respondent reported (1) ever having had anal sexual intercourse with a man (ACASI; 257 men), (2) having had at least 1 male partner during the past 12 months (ACASI; 164 men), and (3) having had a heterosexual partnership that spanned the entire 12 months preceding the interview (CAPI; 24 men).

\section{Missing Data, Exclusions, and Logic Checks}

When the date of first or last sexual intercourse with a partner was missing, we estimated the date from the respondent's age at first or last sexual intercourse with that partner or used dates of marriage, cohabitation, or separation. These imputations were needed for less than $1 \%$ of dates of first and last sexual intercourse for partnerships reported in the CAPI questionnaire. Because we sought to determine the prevalence of concurrency during the preceding year, sexual partnerships that ended more than 12 months before the interview were excluded from the analyses. We visually reviewed sexual partnership histories, characteristics, and other information of the 65 respondents who reported 2 or more sexual partnerships with identical starting and ending dates, and excluded 31 sexual partnerships from analysis because they appeared to represent duplicate reports of the same partnership.

\section{Estimation of Overall Concurrency Prevalence}

In both the CAPI and ACASI sections of the interview, respondents were asked how many women they had had sexual intercourse with during the past year, although with differently worded questions. Substantial agreement existed between CAPI and ACASI (91\%; $\kappa=0.79 ; 95 \%$ confidence interval $[\mathrm{CI}]=0.75,0.81$ ) in respondents' reports of whether or not they had multiple female partners. Men tended to report more partners in ACASI than in CAPI, consistent with the concept that ACASI increases disclosure of sensitive behavior ${ }^{13}$-but also possibly a reflection of the inclusion of oral sexual intercourse in the ACASI definition of sexual intercourse. Concurrency could not be directly determined from the ACASI section because it did not elicit partnership dates.

To account for possible underreporting of concurrent sexual partners, we estimated the population prevalence of concurrency by applying the concurrency prevalence among men who reported the same number of sexual partners in both the CAPI and ACASI sections to all men who reported that number of sexual partners in ACASI. For example, because concurrency prevalence was 38\% among men who reported 2 sexual partners during the past year in both CAPI and ACASI, we estimated the overall prevalence by assuming a concurrency prevalence of $38 \%$ among the 227 men who reported 2 sexual partners in ACASI but had data for fewer than 2 sexual partners in CAPI. In our results, we report overall concurrency prevalence estimated with and without this adjustment for underreporting.

\section{Correlates of Concurrency}

The adjustment for underreporting was used only for overall prevalence estimates. Analyses of individual-level associations with concurrency were on the basis of concurrency status as determined only by sexual partnership dates (and information concerning sexual intercourse with men). We examined the association between concurrent sexual partnerships with variables found to be associated with concurrency in previous studies: age at interview, marital status, education, employment, annual household income in the 12 months prior to the survey (as a percentage of the federal poverty limit), age at first sexual intercourse, history of incarceration, sexual activity with another man, substance use, drug or alcohol intoxication during sexual activity, and sexually transmitted infection. For men 22 years and older, we examined poverty, employment, and education as indicators of socioeconomic status.

We examined associations separately among non-Hispanic Whites, non-Hispanic Blacks, and Hispanics. The survey included too few men of other racial/ethnic groups to permit separate analyses of concurrency among them, but these men were included in analyses of the total population. We fit multiple logistic models with concurrency as the dependent variable in a restricted data set (men 22 years and older who had been sexually active for at least 1 year), as in our previous NSFG analysis. ${ }^{10}$ Variables with multiple levels (age, age at first sexual intercourse, education, income) were analyzed as unordered categorical variables. Independent variables were removed from the model if they were not associated with concurrency and their removal did not change the coefficients of other variables by more than $10 \%$ (in fact, no coefficients changed by more than $5 \%$ ). All analyses were weighted using the variable provided in the NSFG data set (FINALWGT). All analyses were conducted with the survey sampling commands in Stata version 9.0 (Stata Corp, College Station, Texas) to account for the survey's complex sampling design.

\section{RESULTS}

\section{Demographic Characteristics}

Familiar ethnic disparities in socioeconomic resources were evident (Table 1): compared with non-Hispanic White men, non-Hispanic Black, Hispanic, and men of other racial/ ethnic groups were much less likely to have a college degree, report annual incomes greater than $150 \%$ of the federal poverty line, or own their own home. Non-Hispanic Black, Hispanic, and men of other racial/ethnic groups were also much more likely than nonHispanic White men to have spent a night in a shelter within the past year.

\section{Sexual Relationship History}

Non-Hispanic Black men were substantially less likely to be married at the time of the interview (31\%) than were non-Hispanic White men (44\%), Hispanic men (43\%), and men from other racial/ethnic backgrounds (40\%; Table 2). Half of non-Hispanic Black men (51\%) had never married. 
TABLE 1-Sample Demographic Characteristics, by Race/Ethnicity: US Men, National Survey of Family Growth, 2002

\begin{tabular}{|c|c|c|c|c|c|}
\hline & $\begin{array}{l}\text { Non-Hispanic } \\
\text { White }\end{array}$ & $\begin{array}{l}\text { Non-Hispanic } \\
\text { Black }\end{array}$ & Hispanic & Other & Total \\
\hline Unweighted no. & 2601 & 930 & 1123 & 274 & 4928 \\
\hline Weighted \% & 65.4 & 11.9 & 16.7 & 6.1 & 100 \\
\hline \multicolumn{6}{|l|}{ Age, $y, \%$} \\
\hline $15-17$ & 9.3 & 12.2 & 8.4 & 8.2 & 9.4 \\
\hline $18-22$ & 16.9 & 18.5 & 19.0 & 16.8 & 17.5 \\
\hline $23-24$ & 5.4 & 6.8 & 7.8 & 6.5 & 6.0 \\
\hline $25-29$ & 13.7 & 14.4 & 19.0 & 20.4 & 15.1 \\
\hline $30-34$ & 16.6 & 15.4 & 17.6 & 15.7 & 16.6 \\
\hline $35-39$ & 18.0 & 16.1 & 15.4 & 16.9 & 17.3 \\
\hline $40-45$ & 20.1 & 16.7 & 12.9 & 15.5 & 18.2 \\
\hline \multicolumn{6}{|l|}{ Nativity, \% } \\
\hline US born & 96.0 & 89.9 & 46.3 & 57.1 & 84.6 \\
\hline Foreign born & 4.0 & 10.1 & 53.7 & 42.9 & 15.4 \\
\hline \multicolumn{6}{|l|}{ Work status, ${ }^{a} \%$} \\
\hline Full-time & 75.7 & 67.9 & 72.4 & 60.6 & 73.3 \\
\hline Part-time & 12.2 & 16.4 & 16 & 16.2 & 13.5 \\
\hline Unemployed & 9.2 & 11.3 & 9 & 21.9 & 10.2 \\
\hline Other & 3.0 & 4.4 & 2.6 & 1.3 & 3.0 \\
\hline \multicolumn{6}{|l|}{ Education, ${ }^{a} \%$} \\
\hline$<$ High school & 8.8 & 12.6 & 34.7 & 9.1 & 13.5 \\
\hline High school diploma/GED & 32.1 & 44.4 & 34.6 & 22.6 & 33.3 \\
\hline Some college & 29.2 & 29.6 & 20.1 & 30.7 & 27.9 \\
\hline Bachelors degree or higher & 29.9 & 13.5 & 10.6 & 37.7 & 25.3 \\
\hline \multicolumn{6}{|l|}{ Household income as a $\%$ of 2000 poverty line ${ }^{a}$} \\
\hline$<150 \%$ & 14.2 & 28.9 & 37.3 & 31.6 & 20.7 \\
\hline $150 \%-249 \%$ & 16.0 & 21.8 & 27.4 & 27.5 & 19.2 \\
\hline $250 \%-399 \%$ & 28.1 & 26.1 & 21.6 & 15.5 & 26.0 \\
\hline$\geq 400 \%$ & 41.7 & 23.2 & 13.7 & 25.4 & 34.0 \\
\hline \multicolumn{6}{|l|}{ Residence, ${ }^{\mathrm{a}} \%$} \\
\hline Owned house & 63.9 & 42.7 & 35.3 & 39.2 & 55.3 \\
\hline Rented & 36.1 & 57.3 & 64.8 & 60.8 & 44.8 \\
\hline Spent night in shelter in past 12 months & 1.1 & 3.6 & 3.0 & 3.5 & 1.9 \\
\hline
\end{tabular}

Notes. GED = general equivalency diploma. Estimates are weighted percentages.

${ }^{\mathrm{a} E x c l u d e s ~ m e n ~ y o u n g e r ~ t h a n ~} 22$ years.

More than half (59\%) of men first had sexual intercourse by age 16 or 17 years. NonHispanic Black men initiated sexual intercourse earlier than did men in other racial/ ethnic groups ( $49 \%$ by age 14 or 15 compared with $25 \%$ of non-Hispanic White men and $35 \%$ of Hispanic men). Non-Hispanic Black men also reported more sexual partners during their lifetime; $30 \%$ had more than 10 sexual partners compared with $24 \%$ of
non-Hispanic White men, 18\% of Hispanic men, and $13 \%$ of men of other race/ ethnicities. During the preceding year most men in all ethnic groups had no more than 1 sexual partner, but the prevalence of multiple sexual partnerships varied substantially by race/ethnicity. Non-Hispanic Black and Hispanic men ( $28 \%$ and $18 \%$, respectively) were more likely to have had multiple sexual partners than were non-Hispanic White (13\%) and men of other racial/ethnic groups (9\%). Relatively few men (2\%) reported having exchanged sexual intercourse for drugs or money. A total of $6 \%$ of men reported ever having had oral or anal sexual intercourse with a man, and 3\% of men had at least 1 male sexual partner in the past year.

\section{Substance Use and Sexually Transmitted Infections}

Non-Hispanic White men were more likely (36\%) to report drug or alcohol intoxication while having sexual intercourse during the past year than were men of other racial/ ethnic groups (non-Hispanic Black, 30\%; Hispanic, 28\%; Other, 21\%). Non-Hispanic White (28\%) and Hispanic (25\%) men were more likely than non-Hispanic Black (22\%) and men of other racial/ethnic groups (16\%) to report binge alcohol consumption at least monthly. No differences by race/ethnicity were found in crack or cocaine use or frequent marijuana use during the past year.

Non-Hispanic Black men were more likely than non-Hispanic White or Hispanic men to have received medication for a sexually transmitted infection (5\%) within the past year. Among men who had more than 1 sexual partner in the past year, a larger proportion of non-Hispanic Black men (58\% [vs 45\% for all men]) used condoms during the last sexual intercourse with all of their partners.

\section{Prevalence of Concurrent Sexual Partnerships}

The prevalence of concurrency with no adjustment was $6.6 \%(95 \% \mathrm{CI}=5.8 \%$, $7.4 \%)$. Overall concurrency prevalence estimates, adjusted for missing dates of female sexual partnerships, were $11 \%$ among all men, 12\% among sexually experienced men, and $12.5 \%$ among men who had at least 1 sexual partner in the past year. Although most concurrent sexual partnerships involved only female partners, among the 164 men with male sexual partners in the past year, 40 (35\%, weighted) reported ever having had sexual intercourse with a woman, and concurrency in the past year was identified among 24 men who met our definition of concurrency (see "Methods" section) because of apparent overlap between same-sex and heterosexual partnerships. 
TABLE 2-Sexual Relationship History and Other Risk Factors Among US Men, by Race/Ethnicity: National Survey of Family Growth, 2002

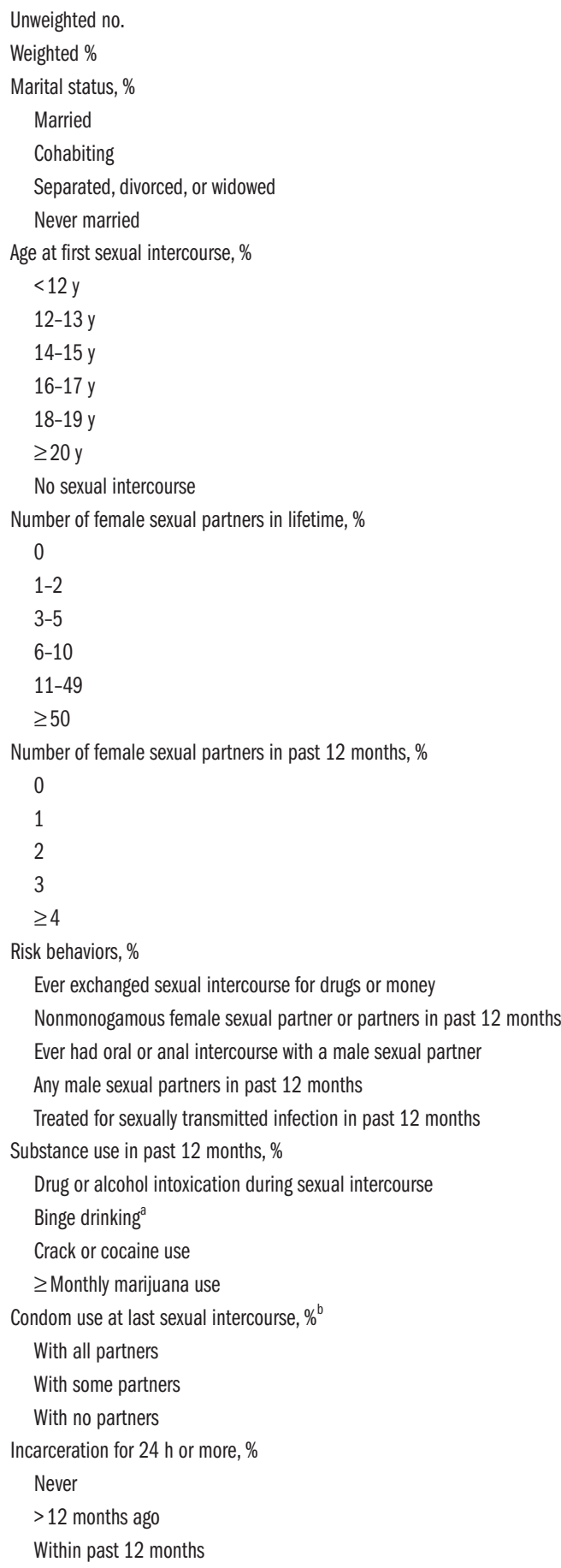

Drug or alcohol intoxication during sexual intercourse $\quad 36.2$

\begin{tabular}{ccccr}
$\begin{array}{c}\text { Non-Hispanic } \\
\text { White }\end{array}$ & $\begin{array}{c}\text { Non-Hispanic } \\
\text { Black }\end{array}$ & Hispanic & Other & Total \\
\hline 2601 & 930 & 1123 & 274 & 4928 \\
65.4 & 11.9 & 16.7 & 6.1 & 100
\end{tabular}

44.4

7.8

7.5

40.3

1.3

4.7

18.6

31.2

17.2

14.1

12.9

\section{7}

19.8

20.8

21.8

19.8

4.0

21.7

64.9

7.4

2.7

3.4

1.5

9.5

6.4

2.8

1.9

28.0

7.5

14.3

40.3

33.3

26.3

74.6

20.4

5.0

\section{8}

9.9

8.2

51.0

6.8

15.4

26.9

26.6

9.2

5.5

9.7

9.9

14.2

20.0

26.2

24.8

5.0

17.1

55.1

11.0

7.9

8.9

4.3

17.8

5.5

3.5

5.2

30.1

21.9

4.6

14.2

57.6

32.3

10.0

67.8

19.7

12.5

42.7

14.0

5.2

38.1

2.5

9.0

$$
23.4
$$

$$
28.2
$$

$$
15.5
$$

$$
11.6
$$$$
9.9
$$

$$
10.0
$$

$$
24.3
$$

$$
27.6
$$$$
20.6
$$$$
15.8
$$$$
1.8
$$

$$
\begin{aligned}
& 17.6 \\
& 64.6
\end{aligned}
$$$$
8.1
$$$$
5.2
$$$$
4.5
$$

40.2

$$
10.0
$$$$
4.1
$$$$
45.5
$$

42.2

9.2

7.0

41.6

21

6.7

20.1

30.0

30.0
15.5

13.2

12.4

12.9

20.2

21.8

22.3

19.3

Note. Estimates are weighted percentages.

${ }^{a}$ Consumed at least 5 drinks in 1 day on 5 or more occasions in past 12 months.

${ }^{b}$ Restricted to men who reported sexual partnership dates from at least 2 sexual partners during the past year. 
TABLE 3-Unadjusted Prevalence Odds Ratios (ORs) and Crude Prevalence ORs for Concurrent Sexual Partnerships Among US Men, by Race/Ethnicity: National Survey of Family Growth, 2002

\begin{tabular}{|c|c|c|c|c|}
\hline & $\begin{array}{l}\text { Non-Hispanic White, } \\
\text { OR (95\% CI) }\end{array}$ & $\begin{array}{l}\text { Non-Hispanic Black, } \\
\text { OR }(95 \% \mathrm{Cl})\end{array}$ & $\begin{array}{l}\text { Hispanic, } \\
\text { OR (95\% Cl) }\end{array}$ & $\begin{array}{c}\text { Total, } \\
\text { OR (95\% Cl) }\end{array}$ \\
\hline Unweighted no. & 2601 & 930 & 1123 & $4928^{\mathrm{a}}$ \\
\hline \multicolumn{5}{|c|}{ Unadjusted Prevalence Odds Ratios } \\
\hline \multicolumn{5}{|l|}{ Current age, y } \\
\hline $15-17$ & $0.48(0.17,1.32)$ & $0.54(0.21,1.38)$ & $1.33(0.45,3.95)$ & $0.71(0.38 .1 .32)$ \\
\hline $18-22$ & $2.16(1.03,4.55)$ & $1.82(0.87,3.78)$ & $2.38(0.97,5.85)$ & $2.19(1.32,3.61)$ \\
\hline $23-24$ & $3.03(1.31,7.05)$ & $2.52(0.99,6.44)$ & $2.96(0.90,9.76)$ & $3.25(1.82,5.82)$ \\
\hline $25-29$ & $1.27(0.53,3.03)$ & $1.01(0.46,2.23)$ & $1.72(0.69,4.25)$ & $1.52(0.86,2.67)$ \\
\hline $30-34$ & $0.95(0.39,2.30)$ & $1.58(0.67,3.7)$ & $1.76(0.67,4.63)$ & $1.27(0.72,2.24)$ \\
\hline $35-39$ & $0.86(0.39,1.89)$ & $0.93(0.44,1.98)$ & $0.57(0.19,1.76)$ & $0.86(0.50,1.47)$ \\
\hline \multicolumn{5}{|l|}{ 40-44 (Ref) } \\
\hline \multicolumn{5}{|l|}{ Race/ethnicity } \\
\hline \multicolumn{5}{|l|}{ Non-Hispanic White (Ref) } \\
\hline Non-Hispanic Black & & & & $3.06(2.27,4.13)$ \\
\hline Hispanic & & & & $1.69(1.22,2.35)$ \\
\hline Other & & & & $0.88(0.37,2.08)$ \\
\hline \multicolumn{5}{|l|}{ Household income as a \% of 2000} \\
\hline \multicolumn{5}{|l|}{ poverty line $\mathrm{e}^{\mathrm{b}}$} \\
\hline \multicolumn{5}{|l|}{$<150 \%$ (Ref) } \\
\hline $150 \%-249 \%$ & $0.63(0.18,2.29)$ & $0.97(0.41,2.26)$ & $0.83(0.34,1.98)$ & $0.64(0.34,1.19)$ \\
\hline $250 \%-399 \%$ & $0.85(0.39,1.83)$ & $0.67(0.32,1.4)$ & $1.05(0.48,2.33)$ & $0.71(0.45,1.13)$ \\
\hline$\geq 400 \%$ & $1.20(0.59,2.43)$ & $1.79(0.92,3.48)$ & $3.71(1.68,8.18)$ & $1.14(0.75,1.73)$ \\
\hline Foreign-born & $1.75(0.45,6.75)$ & $0.84(0.42,1.67)$ & $0.49(0.28,0.84)$ & $0.89(0.58,1.38)$ \\
\hline \multicolumn{5}{|l|}{ Education $^{\mathrm{b}}$} \\
\hline \multicolumn{5}{|l|}{ Bachelors degree or higher (Ref) } \\
\hline Some college & $1.86(0.99,3.48)$ & $0.67(0.30,1.50)$ & $1.18(0.52,2.69)$ & $1.75(1.13,2.7)$ \\
\hline High-school diploma/GED & $1.10(0.59,2.06)$ & $0.85(0.42,1.72)$ & $0.51(0.21,1.24)$ & $1.24(0.83,1.87)$ \\
\hline$<$ High school & $3.50(1.4,8.76)$ & $0.85(0.37,2.00)$ & $0.55(0.21,1.44)$ & $2.02(1.14,3.55)$ \\
\hline \multicolumn{5}{|l|}{ Work status ${ }^{b}$} \\
\hline \multicolumn{5}{|l|}{ Full-time (Ref) } \\
\hline Part-time & $1.69(0.90,3.17)$ & $1.14(0.55,2.39)$ & $0.98(0.45,2.13)$ & $1.31(0.86,1.99)$ \\
\hline Unemployed & $2.57(1.27,5.18)$ & $1.91(0.83,4.41)$ & $1.32(0.59,2.95)$ & $1.86(1.16,3.01)$ \\
\hline Other & $0.96(0.27,3.38)$ & $0.92(0.24,3.48)$ & $4.32(0.65,28.73)$ & $1.48(0.55,3.97)$ \\
\hline \multicolumn{5}{|c|}{ Crude Prevalence Odds Ratios } \\
\hline \multicolumn{5}{|l|}{ Marriage status } \\
\hline \multicolumn{5}{|l|}{ Married (Ref) } \\
\hline Cohabiting & $3.51(1.05,11.77)$ & $4.23(1.58,11.29)$ & $0.95(0.31,2.88)$ & $2.81(1.38,5.70)$ \\
\hline Separated, divorced, or widowed & $6.73(2.53,17.90)$ & $9.70(3.80,24.77)$ & $8.80(3.42,22.66)$ & $6.95(3.81,12.67)$ \\
\hline Never married & $7.36(2.95,18.39)$ & $7.30(3.34,15.96)$ & $4.98(2.40,10.32)$ & $6.20(3.62,10.62)$ \\
\hline \multicolumn{5}{|l|}{ Age at first sexual intercourse, $y$} \\
\hline \multicolumn{5}{|l|}{20 (Ref) } \\
\hline $18-19$ & $4.59(1.71,12.35)$ & $1.38(0.25,7.65)$ & $1.76(0.49,6.30)$ & $3.47(1.71,7.07)$ \\
\hline $16-17$ & $5.16(2.09,12.78)$ & $3.53(0.85,14.71)$ & $1.96(0.64,6.04)$ & $4.12(2.19,7.72)$ \\
\hline $14-15$ & $9.25(3.73,22.92)$ & $8.86(2.18,35.98)$ & $4.67(1.58,13.83)$ & $9.78(5.26,18.19)$ \\
\hline $12-13$ & $7.82(2.68,22.87)$ & $9.18(2.17,38.77)$ & $5.31(1.49,18.89)$ & $10.32(5.23,20.38)$ \\
\hline$<12$ & $8.79(2.05,37.61)$ & $12.59(2.68,59.19)$ & $1.87(0.32,10.94)$ & $12.11(5.32,27.57)$ \\
\hline
\end{tabular}

Correlates of Concurrent Partnerships

Univariate analyses. The odds of concurrency (as estimated without imputation) were highest in the young adult years (age 18-24 years; Table 3). Marked racial/ethnic differences in concurrency were evident. Compared with non-Hispanic White men, concurrency was more likely to occur among non-Hispanic Black men (odds ratio $[\mathrm{OR}]=3.06$ ) and Hispanic men $(\mathrm{OR}=1.69)$. These ethnic patterns persisted, with minor exceptions, in stratified analyses across all variables. Concurrency was associated with the highest income level among non-Hispanic Black men $(\mathrm{OR}=1.79)$ and strongly so for Hispanic men $(\mathrm{OR}=3.71)$.

Foreign-born Hispanic men were considerably less likely than US-born Hispanic men to have concurrent sexual partnerships ( $\mathrm{OR}=$ 0.49). Education and employment status were related to concurrency only among nonHispanic White men: Men without a highschool education were more likely $(\mathrm{OR}=3.50)$ to have concurrent sexual partnerships than men with at least a bachelors degree.

Concurrency was much more likely to have occurred among men who were previously married $(\mathrm{OR}=6.95)$ or never married $(\mathrm{OR}=6.20)$ than among those who were currently married. Concurrency was strongly associated with early age at first sexual intercourse; the odds of concurrency were highest for men who first had sexual intercourse at a young age (ORs were 9.78, 10.32, and 12.11, respectively, for those who had first sexual intercourse at age 14-15, 12-13, and younger than 12 years, compared with men who had sexual intercourse for the first time after age 20 years).

Men with concurrent sexual partnerships were less likely than men without concurrency to have used condoms during last sexual intercourse. Concurrent sexual partnerships were much more likely among men who had a female partner whom they believed had concurrent sexual partners $(\mathrm{OR}=13.00)$ or those who ever had anal sexual intercourse with a woman $(\mathrm{OR}=2.63)$ compared with men who did not have these characteristics. Concurrent sexual partnerships were also more likely among the men who reported having had sex with a man $(\mathrm{OR}=3.09$ if during past year) and among men who had been 
TABLE 3-Continued

\begin{tabular}{|c|c|c|c|c|}
\hline \multicolumn{5}{|l|}{ Condom u } \\
\hline \multicolumn{5}{|l|}{ intercourse $^{c}$} \\
\hline \multicolumn{5}{|l|}{ With all partners (Ref) } \\
\hline With some partners & $1.05(0.57,1.92)$ & $1.94(0.88,4.26)$ & $2.37(1.00,5.61)$ & $1.31(0.87,2.00)$ \\
\hline With no partners & $3.35(1.83,6.11)$ & $1.97(0.70,5.51)$ & $2.00(0.73,5.42)$ & $2.27(1.45,3.56)$ \\
\hline \multicolumn{5}{|l|}{ Risk behaviors } \\
\hline $\begin{array}{l}\text { Nonmonogamous female sexual } \\
\text { partners in past } 12 \text { months }\end{array}$ & $13.23(8.57,20.44)$ & $11.18(6.88,18.18)$ & $10.18(5.75,18.02)$ & $13.00(9.74,17.36)$ \\
\hline $\begin{array}{l}\text { Ever had anal sexual intercourse } \\
\text { with female }\end{array}$ & $2.62(1.71,4.02)$ & $3.66(2.36,5.69)$ & $1.86(1.12,3.09)$ & $2.63(2.01,3.45)$ \\
\hline $\begin{array}{l}\text { Any male sexual partners in past } \\
12 \text { months }\end{array}$ & $2.00(0.88,4.53)$ & $2.68(0.84,8.62)$ & $5.15(2.20,12.05)$ & $3.09(1.82,5.22)$ \\
\hline $\begin{array}{l}\text { Ever had oral or anal intercourse } \\
\text { with a male sexual partner }\end{array}$ & $1.97(1.03,3.77)$ & $2.17(0.88,5.37)$ & $2.97(1.47,6.02)$ & $2.25(1.47,3.46)$ \\
\hline \multicolumn{5}{|l|}{ Incarceration for $24 \mathrm{~h}$ or more } \\
\hline \multicolumn{5}{|l|}{ Never (Ref) } \\
\hline$>12$ months ago & $1.90(1.17,3.07)$ & $2.07(1.23,3.49)$ & $0.88(0.48,1.63)$ & $1.82(1.31,2.51)$ \\
\hline Within past 12 months & $5.80(3.27,10.3)$ & $1.96(0.99,3.86)$ & $3.00(1.48,6.08)$ & $4.26(2.94,6.16)$ \\
\hline \multicolumn{5}{|l|}{ Substance use in past 12 months } \\
\hline Binge alcohol use ${ }^{d}$ & $3.04(2.01,4.59)$ & $2.18(1.31,3.62)$ & $2.00(1.20,3.36)$ & $2.43(1.87,3.17)$ \\
\hline Monthly marijuana use & $3.09(2.00,4.78)$ & $2.47(1.38,4.42)$ & $2.59(1.37,4.89)$ & $2.72(2.01,3.69)$ \\
\hline Crack or cocaine use & $4.86(2.97,7.95)$ & $3.88(1.42,10.61)$ & $2.01(0.91,4.42)$ & $3.39(2.32,4.95)$ \\
\hline $\begin{array}{l}\text { Drug or alcohol intoxication } \\
\text { during sexual intercourse }\end{array}$ & $6.06(3.59,10.24)$ & $3.42(2.19,5.34)$ & $2.96(1.76,4.98)$ & $4.19(3.16,5.55)$ \\
\hline
\end{tabular}

Note. GED = general equivalency diploma; $\mathrm{Cl}=$ confidence interval. Estimates are prevalence odds ratios from the unadjusted concurrency prevalence.

${ }^{a}$ Men in the Other race/ethnicity category are included in the total.

${ }^{\mathrm{b}}$ Among respondents 22 years and older: unweighted numbers are 1766 for non-Hispanic Whites, 676 for non-Hispanic Blacks, 807 for Hispanics (total; $\mathrm{N}=3435$ ).

${ }^{\mathrm{C}}$ Restricted to men who reported sexual partnership dates from at least 2 sexual partners during the past year.

${ }^{d}$ Consumed at least 5 drinks in 1 day on 5 or more occasions in past 12 months.

incarcerated $(\mathrm{OR}=4.26$ if incarcerated within the past year, $\mathrm{OR}=1.82$ if incarcerated more than 1 year ago). Concurrency was associated with substance use, including frequent binge alcohol consumption $(\mathrm{OR}=2.43)$, frequent marijuana use $(\mathrm{OR}=2.72)$, crack cocaine use during the past year $(\mathrm{OR}=3.39)$, and drug or alcohol intoxication while having sexual intercourse during the past year $(\mathrm{OR}=4.19)$.

\section{Multiple Logistic Models}

The associations between concurrent sexual partnerships and race/ethnicity, higher income, marital status, earlier age at first sexual intercourse, alcohol or drug intoxication during sexual intercourse within the past year, incarceration within the past year, having a nonmonogamous female partner, and history of sexual intercourse with a man remained in multiple logistic models. Associations between concurrency and age, education, employment, age at first sexual intercourse, and frequent alcohol consumption were not significant, and their removal did not meaningfully change the values of the ORs for the other covariates. Use of crack or cocaine, frequent binge alcohol consumption, and frequent marijuana use were removed from the model because they were not associated with concurrency in the final model with or without the variable "intoxication during sex." Moreover, each of these variables (crack, alcohol, and marijuana) was associated with the variable "intoxication during sex," which was retained in the multivariable model. Odds ratios for the final model are presented in Table 4.

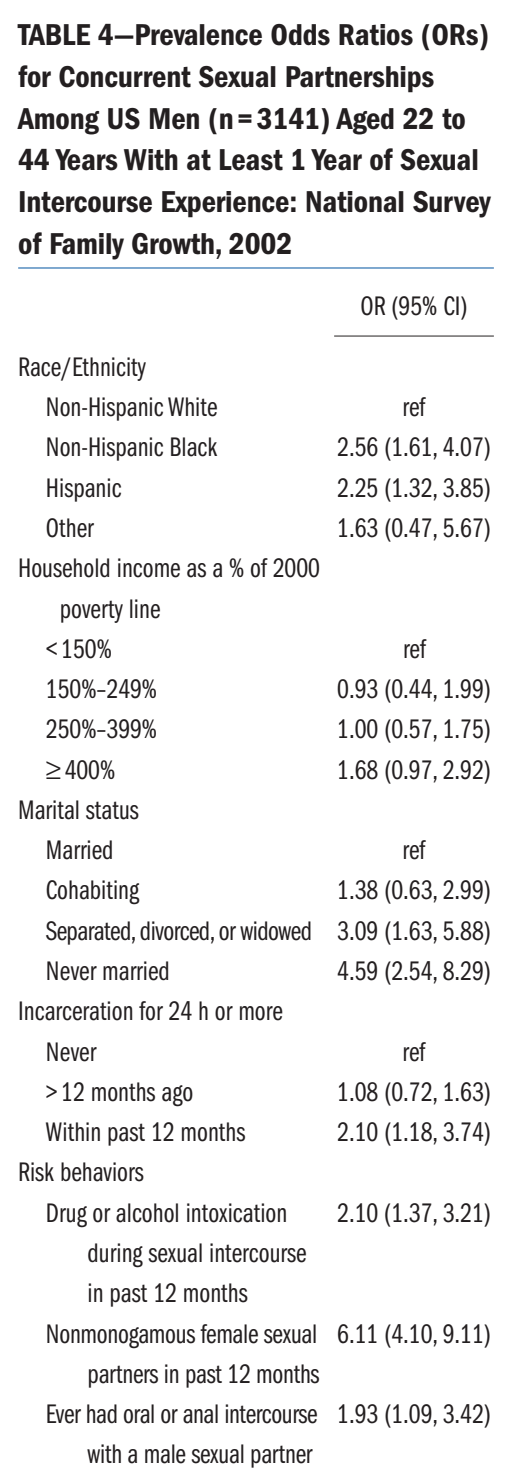

Notes. $\mathrm{Cl}=$ confidence interval. ORs were estimated from multiple logistic regression models for concurrency prevalence, derived without data from the audio computer-assisted self-interviewing on the number of female sexual partners (see "Methods" section for information on the audio computerassisted self-interview).

\section{DISCUSSION}

In this nationally representative sample, the prevalence of concurrent sexual partnerships among US men was about 11\% during the preceding year. Concurrency was associated with several demographic variables: being unmarried, non-Hispanic Black or Hispanic race/ ethnicity, and incarceration during the past year. Men with concurrent sexual partnerships 
were also more likely than those without concurrent sexual partnerships to report several risk behaviors, including drug or alcohol intoxication during sexual intercourse, nonmonogamous female sexual partners, and history of ever having had sexual intercourse with a man.

Relatively few data have been published concerning the extent of concurrent sexual partnerships among men. A population-based survey reported that $15 \%$ of men in Britain aged between 16 and 44 years had concurrent sexual partnerships in the past year. ${ }^{14}$ In a random-digit-dialing survey of young adults in Seattle, about $21 \%$ of men had a concurrent sexual partnership during their most recent relationship, but prevalence of concurrency during the past year was not reported. ${ }^{15}$

Several individual correlates of concurrency have been identified. As expected, concurrency was strongly associated with being unmarried, a finding that other studies (including those among women) have noted as well. ${ }^{10,15}$ Compared with those who were unmarried, married people were far more likely to have only 1 sexual partner and, thus, less likely to have concurrent sexual partnerships. ${ }^{16}$ The association between concurrency and younger age,${ }^{10}$ especially adolescence, ${ }^{17,18}$ has also been observed in the past.

Several reports have noted an association between incarceration and concurrency. ${ }^{15,19-21}$ In our study, men who had been incarcerated were more likely to have concurrent sexual partnerships than were those who had never been incarcerated. Non-Hispanic Black men and women in rural North Carolina whose sexual partner had been incarcerated were also more likely to have concurrent sexual partnerships. ${ }^{19,20}$ Incarceration may be a marker for high-risk behaviors and characteristics that destabilize long-term partnerships, but imprisonment can also promote concurrency by physically removing 1 partner and, thus, disrupting existing relationships. Moreover, the decreased employment prospects and poverty that often result from incarceration hinder long-term partnerships as well. ${ }^{22-25}$ Given its high prevalence in the United States, incarceration is a potentially important distal factor in sexual transmission of HIV infection through an effect on concurrency. ${ }^{22,21}$

The higher prevalence of concurrent sexual partnerships among non-Hispanic Black and Hispanic men may contribute to the high rates of heterosexually transmitted HIV infection among non-Hispanic Black and Hispanic women in the United States. Having a sexual partner with concurrent sexual partnerships was an individual-level risk factor for heterosexually transmitted HIV infection among non-Hispanic Black men and women without high-risk behavior. ${ }^{9}$

Several other findings are of concern because of their influence on sexual networks and resultant disease transmission. First, concurrency was associated in crude analyses with frequent substance use and in multivariable analyses with drug or alcohol intoxication while having sexual intercourse, a finding that suggests coupling of the elevated risk from substance use with the transmission potential of concurrency. Substance use, especially crack cocaine use, is in fact associated with concurrent sexual partnerships among non-Hispanic Black men and women with heterosexually transmitted HIV infection. ${ }^{19}$

Second, the observation that men who had concurrent sexual partnerships with women were more likely to have also had sexual intercourse with men indicates bridging between heterosexual women and men who have sex with men, a network pattern that enhances HIV transmission throughout the population. Third, men with concurrent sexual partnerships were more likely than those without such partnerships to report that their female sexual partners also had concurrent sexual partners. This finding has been noted in populations with high HIV rates ${ }^{19,20}$ and suggests the existence of dense sexual networks that facilitate the spread of HIV infection. ${ }^{26}$

A major strength of this study is its use of a large, high-quality, nationally representative data set that oversamples non-Hispanic Blacks and Hispanics. Limitations include absence of specific information concerning condom use during male same-sex interactions, and those limitations expected with survey data, including the possibility that nonrespondents differ meaningfully from respondents in ways that are not removed by nonresponse adjustments to sample weights, reliance on disclosure of embarrassing information, and the need for respondents to recall months of first and last sexual intercourse with different partners during the preceding year.
The differences in sexual partner number distribution for the CAPI and ACASI interviews illustrate the difficulty of obtaining accurate data on sexual partnerships, and our imputation method has not been validated for this specific use. Our analysis is likely to have slightly underestimated the prevalence of concurrency from the CAPI data among the $4 \%$ of respondents with more than 3 sexual partners during the preceding year, because the NSFG did not attempt to obtain dates for more than 3 recent sexual partners.

We estimate that $11 \%$ of US men had concurrent sexual partnerships during the 12 months covered by the NSFG Cycle 6 . The higher prevalence in various groups and indications of dense sexual networks, mixing between high-risk subpopulations, and mixing between high-risk subpopulations and the general population may be important factors in the epidemiology of heterosexual HIV infection in the United States and in continuing epidemic transmission in the non-Hispanic Black and Hispanic populations. ${ }^{27}$

\section{About the Authors}

Adaora A. Adimora and Victor J. Schoenbach are with the School of Public Health, University of North Carolina, Chapel Hill. Adaora A. Adimora and Irene A. Doherty are with the School of Medicine, University of North Carolina, Chapel Hill.

Requests for reprints should be sent to Adaora A. Adimora, MD, MPH, Division of Infectious Diseases, 130 Mason Farm Rd, CB \#7030, Bioinformatics Building, UNC School of Medicine, Chapel Hill, NC 275997030 (e-mail: adimora@med.unc.edu).

This article was accepted February 14, 2007

\section{Contributors}

A.A. Adimora and V.J. Schoenbach originated the study and supervised all aspects of its implementation. I. A. Doherty completed the analyses.

\section{Acknowledgments}

This work was supported by the National Institute of Allergy and Infectious Diseases (to A.A. Adimora; award 1K02 AI01867-01) and the National Institute of Child Health and Human Development (to A.A. Adimora; award 1 R21 HD054293-01A1)

We thank Willard Cates, MD, MPH, for his insightful comments and review of the article.

\section{Human Participant Protection}

This study was exempted from review by the School of Medicine's Office of Human Research Ethics at the University of North Carolina at Chapel Hill. 


\section{References}

1. Aral SO. Sexual network patterns as determinants of STD rates: paradigm shift in the behavioral epidemiology of STDs made visible. Sex Transm Dis. 1999;26: 262-264.

2. Morris M, Kretzschmar M. Concurrent partnerships and transmission dynamics in networks. Soc Networks. 1995;17:299-318.

3. Watts $\mathrm{CH}$, May RM. The influence of concurrent partnerships on the dynamics of HIV/AIDS. Math Biosci. 1992;108:89-104.

4. Anderson RM. Transmission dynamics of sexually transmitted infections. In: Holmes KK, Sparling PF, Mardh P-A, et al., eds. Sexually Transmitted Diseases. 3rd ed. New York, NY: McGraw-Hill; 1999.

5. Pilcher CD, Tien HC, Eron JJ Jr, et al. Brief but efficient: acute HIV infection and the sexual transmission of HIV. J Infect Dis. 2004;189:1785-1792.

6. Doherty IA, Shiboski S, Ellen JM, Adimora AA, Padian NS. Sexual bridging socially and over time: a simulation model exploring the relative effects of mixing and concurrency on viral sexually transmitted infection transmission. Sex Transm Dis. 2006;33: 368-373.

7. Potterat J, Zimmerman-Rogers H, Muth SQ, et al. Chlamydia transmission: concurrency, reproduction number, and the epidemic trajectory. Am J Epidemiol. 1999;150:1331-1339.

8. Koumans E, Farley T, Gibson JJ, et al. Characteristics of persons with syphilis in areas of persisting syphilis in the United States: sustained transmission associated with concurrent partnerships. Sex Transm Dis. 2001;28:497-503.

9. Adimora AA, Schoenbach VJ, Martinson FE, et al Heterosexually transmitted HIV infection among African Americans in North Carolina. J Acquir Immune Defic Syndr. 2006;41:616-623.

10. Adimora A, Schoenbach V, Bonas DM, Martinson FE, Donaldson KH, Stancil TR. Concurrent sexual partnerships among women in the United States. Epidemiology. 2002;13:320-327.

11. National Center for Health Statistics. Cycle 6 NSFG data now available. Vol. 2006. Atlanta, Ga: Centers for Disease Control and Prevention, US Dept of Health and Human Services; 2005. Available at: http://www.cdc.gov/nchs/about/major/nsfg/nsfgcycle6. htm. Accessed February 16, 2006.

12. Groves R, Benson G, Mosher WD, et al. Plan and operation of Cycle 6 of The National Survey of Family Growth. Vital Health Stat 1. 2005;42:1-58

13. Turner CF, Ku L, Rogers SM, Lindberg LD, Pleck JH Sonenstein FL. Adolescent sexual behavior, drug use and violence: increased reporting with computer survey technology. Science. 1998;280:867-873.

14. Johnson AM, Mercer CH, Erens B, et al. Sexual behaviour in Britain: partnerships, practices, and HIV risk behaviours. Lancet. 2001;358:1835-1842.

15. Manhart LE, Aral SO, Holmes KK, Foxman B. Sex partner concurrency: measurement, prevalence, and correlates among urban 18-39-year-olds. Sex Transm Dis. 2002;29:133-143.

16. Laumann EO, Gagnon JH, Michael RT, Michaels S The Social Organization of Sexuality. Chicago, Ill: The University of Chicago Press; 1994:188.
17. Rosenberg MD, Gurvey JE, Adler N, Dunlop MB, Ellen JM. Concurrent sex partners and risk for sexually transmitted diseases among adolescents. Sex Transm Dis. 1999;26:208-212.

18. Ford K, Sohn W, Lepkowski J. American adolescents: sexual mixing patterns, bridge partners, and concurrency. Sex Transm Dis. 2002;29:13-19.

19. Adimora AA, Schoenbach VJ, Martinson FE, Donaldson KH, Stancil TR, Fullilove RE. Concurrent partnerships among rural African Americans with recently reported heterosexually transmitted HIV infection. J Acquir Immune Defic Syndr. 2003;34:423-429.

20. Adimora AA, Schoenbach VJ, Martinson F, Donaldson KH, Stancil TR, Fullilove RE. Concurrent sexual partnerships among African Americans in the rural south. Ann Epidemiol. 2004;14:155-160.

21. Gorbach PM, Stoner BP, Aral SO, Whittington WL Holmes KK. "It takes a village": understanding concurrent sexual partnerships in Seattle, Washington. Sex Transm Dis. 2002;29:453-462.

22. Adimora AA, Schoenbach VJ. Social context, sexual networks, and racial disparities in rates of sexually transmitted infections. J Infect Dis. 2005;191: S115-S122.

23. Ross H, Sawhill I. Time of Transition: The Growth of Families Headed by Women. Washington, DC: The Urban Institute; 1975

24. Butterfield F. Freed from prison, but still paying a penalty: ex-convicts face many sanctions. The New York Times. December 29, 2002;sect A:18.

25. Hoffman S, Holmes J. Husbands, wives, and divorce. In: Duncan G, Morgan J, eds. Five Thousand American Families-Patterns of Economic Progress. Ann Arbor, Michigan: Institute for Social Research; 1976: $23-75$.

26. Potterat J, Rothenberg R, Muth SQ. Network structural dynamics and infectious disease propagation. Int J STD AIDS. 1999;10:182-185.

27. Centers for Disease Control and Prevention. Epidemiology of HIV/AIDS-United States, 1981-2005. MMWR Morb Mortal Wkly Rep. 2006;55:589-592. 